\title{
Germanica
}

\section{Fliegen - Flunkern - Fliehen Literarische Grenzüberschreitungen bei Erica Pedretti, Jürg Laederach, Margrit Baur}

Von Heinz F. Schafroth

\section{CpenEdition}

\section{Journals}

Édition électronique

URL : http://journals.openedition.org/germanica/2496

DOI : 10.4000/germanica.2496

ISSN : 2107-0784

Éditeur

Université de Lille

\section{Édition imprimée}

Date de publication : 30 juin 1990

Pagination : 77-86

ISSN : 0984-2632

Référence électronique

Von Heinz F. Schafroth, «Fliegen - Flunkern - Fliehen Literarische Grenzüberschreitungen bei Erica Pedretti, Jürg Laederach, Margrit Baur », Germanica [Online], 7| 1990, Online erschienen am: 22 Mai 2014, abgerufen am 06 Oktober 2020. URL : http://journals.openedition.org/germanica/2496 ; DOI https://doi.org/10.4000/germanica.2496

Ce document a été généré automatiquement le 6 octobre 2020.

(c) Tous droits réservés 


\title{
Fliegen - Flunkern - Fliehen Literarische Grenzüberschreitungen bei Erica Pedretti, Jürg Laederach, Margrit Baur
}

\author{
Von Heinz F. Schafroth
}

1 Zwei wortwörtliche literarische Umsetzungen des Colloquiumsthemas fallen ein, und es ist schwer vorstellbar, dass sie ihm nicht Pate gestanden haben. Glanzvoll, erhellend genug sind sie beide, Ilse Aichingers Erzählung Meine Sprache und ich ebenso wie der letzte Text von Herta Müllers Prosaband Barfüssiger Februar. Die Autorinnen erzählen beide, wie Sprache die Grenzepassiert: die «kleine Sprache», dit nicht «weitreichende» der Ilse Aichinger, mit «ihrem grauen Blick», die den Zöllnern trotzdem «verdächtig» ist; und das «Minderheitendeutsch» der Herta Müller, «mein Minderheitendeutsch» heisst es, und auch: «mein Schlagabtausch»: «Wie hohlwangig du mir schlägst. Und wenn ich reden will, legst du dich tot auf meine Zunge», so Herta Müller; und Ilse Aichinger: «Wenn meine Sprache die Stimme verliert, hat sie keinen Grund mehr, das Gespräch mit mir sein zu lassen».

2 Grenzüberschreitungen, soviel steht fest, stimmen die Sprache nicht heiter. Sie legt sich quer, wird bockig bis zum Sichtotstellen. Andererseits, und das ist ja auch nicht zu bestreiten: Sprache, jedenfalls diejenige von Literatur, die ihren Namen verdient, wird erst an und jenseits der Grenze virulent, und denen, die mit Sprache umzugehen haben, bleibt gar nichts anderes übrig, als sie immer wieder an die Grenze zu bringen, über die Grenze zu schaffen.

Der Titel dieses Referats ist nicht nur ein Akt der Verliebtheit in die Alliteration. Die drei Infinitive bezeichnen Verhaltensweisen der Autoren gegenüber der Realität. Gemeinsam ist ihnen die in einem weiten Koordinatensystem von Lust, Grimm, Scheu, Bezauberung und Obsession angesiedelte Entschlossenheit, Realität nicht als gegeben, begrenzt und ausschlaggebend $\mathrm{zu}$ verstehen. Sie insistieren auf Erzählund Betrachtungsweisen, die entweder die Grenzen hinter sich lassen oder 
Grenzüberschreitung, Entgrenzung als Utopie des Betrachters und Erzählers bewusstmachen.

4 Verkürzt gesagt: Gemeinsam ist den drei Autoren die nicht realistische Erzählhaltung und -weise; aber auch dies, dass der Nicht-Realismus in keiner Weise eine Spielart der Realitäts/erae ist. Im übrigen visieren die drei Infinitive allerdings keinesfalls eine eingehende Charakterisierung ihres Schreibens an. Sie sollen ausschliesslich fragmentarische Einblicke gewähren 1. in eine Erzählung, 2. in Ausschnitte aus einer solchen, 3. in ein Buch.

\section{Erica Pedrettis Erzählung Über Dittersdorf fliegen'}

5 «Das ist doch nicht meine Hand [...]. Das bin doch nicht ich». Die Erzählung setzt mit gravierenden Zweifeln an der Identität ein. Aber sehr rasch lässt sie die Identität, samt Raum und Zeit und Umständen, die zu ihr gehören, probeweise zu als immerhin mögliche, als allenfalls durchzuspielende: «Zwar könnte ich, ja kann ich mich einmal, probeweise dort hingelen und durchspielen, wie das ist, als eine andere, oder auch warum nicht so wie ich bin? vor Tagesanbruch, im Finstern also, im Bett zu liegen, wach».

Passionierte «Gedankenspiele» (dies, leitmotivisch wiederkehrend, der Ausdruck im Text) heben nun an und lassen Figuren, Landschaften, Bilder, Geschichten oder die Konturen von alledem sich einstellen und wieder verschwinden. Noch ist alles in der Möglichkeitsform belassen, ineinem gleicherweise angstwie lustvollen Irrealis: Angst davor, beim Setzen und Behaupten von erzählerischer Realität ohnehin nur sich selber und der eigenen Geschichte zu begegnen; Lust darauf, andere Identitäten und die Geschichten dieser anderen ausund anzuprobieren, als wären es die eigenen.

7 Danach, der Provisorien müde, entscheidet sich die Erzählung für den Indikativ, erfindet ihr Ich sich «eine ganz andere [...] als das einzig Feststehende», eine Zigeunerin am liebsten, «namens B.». Aber auch «B.» bleibt der Kontrolle unterstellt. Auch ihr wird Sicherheit bei der Behauptung von Realität nicht ohne weiteres durchgelassen. «Ist B. sicher, dass das Fliegen ist?», heisst es, wenn sie auf eine Flugreise geschickt wird: auf einen zunächst richtungsund ziellosen, einen berauschenden und furchteinflössenden Flug, der aber allmählich und immer tiefer in verloren geglaubte, fremdgewordenen Landstriche führt, an den Rand und ins Zentrum von alten Geschichten. Sie spielen in einem abgelegenen Forsthaus und in Zeiten, wo Reisen nicht in Flugzeugen, sondern in Kaleschen unternommen wurden. Doch auch darauf lässt die Erzählung sich nur vorübergehend ein, sie besteht erneut auf dem Fliegen, fliegt auf das Ende der Forsthausgeschichte zu und über das Ende hinaus, und fliegt auch auf ihr, der Geschichte, eigenes Ende zu und darüber hinaus.

Die Metapher «Fliegen» ist im Text vieldeutiger, abgründiger und exakter. Aber noch in der unerlässlichen Verkürzung ist ihre Aussagekraft eminent. Die Metapher braucht bloss beim Wort genommen zu werden. Dann beinhaltet sie nämlich, dass das Erzählen Realität nur noch vermitteln kann als eine, im übertragenen Sinn jetzt, überflogene. Erzählen ist Überfliegen, von vornherein und im nachhinein etwas Unseriöses, Ungehöriges, Unzulässiges also. Nicht etwas Ungenaues allerdings. Ungenau ist zwar die erzählte Realität. Das Überfliegen hingegen, das Erzählen, ist als ein genaues 
vorzustellen: genau in seinen andauernden Perspektiven wechseln, in den Überlagerungen von Räumen, Zeiten, Landschaften, von Personen und Identitäten.

Wirklich deckungsgleich ist es am Ende nicht etwa mit der erzählten Realität, sondern mit sich selber, mit dem ihm eigenen Prinzip des genauen Überfliegens eben. Realität erscheint nur noch aufgelöst in Ansätze dazu, in Fragmente und Segmente, die derweise rasch zuund vorüberfliegen und wieder entschwunden sind, dass kein Anspruch auf Totalität sie je wird einholen können.

Ueberfliegen ermöglicht demnach merkwürdigerweise gerade nicht Uebersicht. Nur flüchtige Einblicke und Eindrückesie allerdings rätselhaft, immer beunruhigend, von allererster Brisanz. «Der Geist lässt das Organ auszucken in Bildern»: Der Satz ist nicht von Erica Pedretti, sondern von Jean Paul, aus Dr. Katzenbergers Badereise, aus dem Kapitel mit dem Titel Die Kunst, einzuschlafen. Dort heisst das, was hier unter dem Begriff des Ueberfliegens zusammengefasst ist, «das wildeste Springen von Gegenzu Gegenstand», das nichts anderes sei als «das [...] Gehenlassen des Gehirns; der Geist lässt das Organ auszucken in Bildern». Das Kapitel endet folgendermassen: «wahrhaftig ich schliefe hier, hielte mich nicht das Schreiben munter, unter demselben ein».

Das Wachsein am Ende von Erica Pedrettis Erzählen ist ein vergleichbares. Vergleichbar ausgesetzt dem Eindringen der Bilder: «Die Bilder dringen mit grösster Schärfe ununterbrochen in sie ein». Zimmerluke, Bordluke: die Standorte des Erzählens sind nicht mehr auseinandergehalten. Ebenso nicht mehr «B.», die erzählte Figur auf ihrem Flug über die Kontinente, und das erzählende Ich, auf einer Matratze, in einem Zimmer liegend: «B. kann nicht aufhören zu schauen, wie ich nicht aufhören kann meine Wahrnehmungen zu formulieren, lautlos, auch ohne Papier». Das ende des Textes Ueber Dittersdorf fliegen ist die endgültige Realisierung der poetologischen Formel: Wer erzählt, überfliegt. Darüber hinaus aber ermöglicht die Ueber fliegensMetapher Einblicke in zentrale Aspekte von Erica Pedrettis Schreiben überhaupt, ist sie erhellend beim Nachdenken über Unverwechselbarkeit und Reiz desselben.

\section{Jürg Laederachs Prosatext «Waldeins Vienna»² ausschnittweise}

«Kann ich mich darauf verlassen, dass Sie den nächsten Text, den Sie auf der Weide treffen, nicht gleich melken wollen wie eine Kuh?». So fragt, in den - wer wollte sie anders nenen! - genialen Grazer Poetik-Vorlesungen, Laederach seine Zuhörer.

Hier und jetzt, in diesem Referat, wird nicht einmal gemolken. Nur gehangelt. Von Unwissen zu Unwissen möglicherweise. Immerhin darf wohl «Unwissenheit» durch «Wissbegierde» ersetzt werden. Mit Laederachs Einverständnis: «Ich rate», sagt er zu den Grazern, und hoffentlich hat es sich so grimmig angehört, wie es soll, «ich rate, wo immer ich es kann, eher zu Wissbegierde als zu Wissen». Der Text Waldeins Vienna beginnt mit einer Ankunft und damit, dass einer seine Arbeit aufnimmt; im fünften Stock eines achtzehnstöckigen verglasten Bürowolkenkratzers, unmittelbar neben dem Dom. Im obersten Stock, im «Fort Knox», lebt Waldein «ein alter Staatstreuer», mit seiner Familie. Einem Sohn beispielsweise, in der Folge einfach «Son» genannt, dessen «einzige schlechte Eigenschaft es war, in aller Höflichkeit und Zuvorkommenheit die Leute anzuspringen, um ihnen die Schienbeine mit Tritten zu brechen». Der son terrorisiert damit die ganze Umgebung, Vater Waldein und eine «mother» 
miteingeschlossen: «Stand einmal die mother des son vor dem son, waren auch ihre Schienbeine gefährdet. Nur stiess er beim Ansprung etwas leisere Schreie aus». Daneben liebt es der son zu spielen. Zum Beispiel mit einer mehrere Etagen hohen Garage. Er lässt Autos, Miniaturausgaben echter Wagen, von der obersten zur untersten Plattform rasen und «wenn ein Miniaturfahrer sich die Knochen brach, kreischte der son vor Vergnügen und Hess gleich einen zweiten Wagen hinten in den ersten hineinfahren».

Zeit wächst der son Vater Waldein über den Kopf, schlägt ihn bei Gelegenheit auch zusammen. Ein Rollentausch findet statt: «es war eine neue PersonenKombination eingetreten. [...] Der son, zum Vater geworden, würde das Auto des zum son gewordenen Vaters Waldein liebevoll zerstören». Es endet mit der Ermordung der ganzen Familie.; und dem Kommentar: «Söhne bringen die eigene Familie um, mit Vorzug die eigene Familie. Es ist einfacher, nicht nur in Vienna, weil die Leute stets bei der Band sind».

«Nicht nur in Vienna», das ist zu beherzigen, für den ganzen Text. Umgekehrt liefert Vienna diesem zweifellos sein einzigartiges Lokalkolorit und somit einen penetranten Geruch von Realität.

An dieser Stelle ist freilich allerhand nachzutragen. Dies zum Beispiel, dass die eben vewendete Formel: «Es endet», angesichts von Laederachs Erzählen eine unverschämte Lüge ist. Es endet viel später, sechzehn Seiten nach dem Vatermord erst, und da nicht weniger lakonisch und zynisch: «Nachdem ich dies alles kennengelernt hatte, reiste ich aus Vienna ab und empfand grosse Reue, bis das nächste Jahr kam». Nicht weniger eine Lüge war es übrigens zu behaupten, Waldeins Vienna beginne mit einer Ankunft. Der Text beginnt vielmehr damit, dass einer von einer zurückliegenden Ankunft erzählt. Ein Ich, das in der Folge im Kreis seiner Generäle, an einer ihm zu Ehren veranstalteten Massenversammlung auf dem Heldenplatz in Wien, die Huldigung eines in Weiss gekleideten Mädchens entgegennimmt.

17 Ueberhaupt war der unternommene, immerhin bald einmal abgebrochene Versuch einer Nacherzählung nichts anderes als eine monumentale Vereinfachung. Es gibt diese halbwegs ordentlichen Stationen des Erzählens gar nicht. Der Text, mit seinen Strukturen des Um-sich-selber-Drehens, der ständigen Repetition und Variation von Formeln und Sätzen, lässt Stationen nicht zu. Und sein Personnal und seine Schauplätze sind nicht nur weit zahlreicher, sondern auch grotesker, verrückter, verzerrter als jede, auch nur ansatzweise versuchte, Nacherzählung es ahnen lässt.

«Immer alles mit allem zu vereinbaren»: danach trachte er, sagt, versichert Laederach in den Vorlesungen. «Das Chaos», fügt er bei, «handelt ähnlich». Waldeins Vienna, das Buch Vor Schrecken starr überhaupt, ist eine exemplarische Umsetzung der chaotischen Handlungsbezw. Erzählweise. Text und Buch sind aber auch die Umsetzung einer andern, wohl nah verwandten Aussage in den Vorlesungen: «Ich kann nur in Regelbrüchen denken, erst stelle ich die Regeln auf, dann verstärke ich sie und mauere sie fest, um sie dann mit umso mehr Kraft brechen zu können».

Aber genau in diesen Sätzen zeigt sich auch (und das ist eine sozusagen vertraueneinflössende Dimension des Textes): es ist darin einer, ein Erzähler eben, am Werk, der souverän, der ein Souverän ist in der Kunst der Regelverletzung: einer, der sie durch und durch beherrscht. Und für seinen aufklärerischen und somit konstruktiven Zynismus gilt in noch höherem Masse das, was Musil für seinen Umgang mit der Ironie beansprucht: dass sie ihm nicht «eine Geste der Ueberlegenheit ist, sondern eine Form 
des Kampfes». Laederachs Zielscheiben sind sehr wohl zu orten, politisch, historisch, psychologisch. Gewalttätigkeit, Faschismus, Rassismus, Heuchelei und anders heissen sie. Oder richtiger gesagt: Bei Laederach heissen sie nicht, sondern sind präsent gerade ohne $\mathrm{zu}$ heissen. Namen und sonstige biographische, historische, psychologische Faktizität ist nicht gefragt, sondern bloss noch und noch subversiv eingeschmuggelt und auf jede Art und Weise herbeigeflunkert.

Dieses Flunkern Laederachis angesichts der Realität ist hemmungslos. Aber es hat sie am Ende systematisch chaotisch eingekreist. Das Flunkern kann demnach nichts Harmloses, nichts Gemütliches, Gemütvolles sein. Eher gleicht es dem lebensrettenden Flunkern des Odysseus. Und das Schreiben dem Abenteuer der gesteuerten Irrfahrt. Nochmals Laederach und die Grazer Vorlesungen: «Ich glaube, mein Wahn, wenn es ein Wahn ist, besteht darin, Literatur [...] als ein Abenteuer anzusehen».

Es bleibe dahingestellt, ob er deswegen der furchteinflössende Autor sein muss, der er für viele noch ist. Obwohl sein Wahn sich gar nicht so grundsätzlich von dem unterscheiden dürfte, der Novalis im Heinrich von Ofterdingen sagen lässt: «Ich weiss nur soviel, dass für mich die Fabel Gesamtwerkzeug meiner gegenwärtigen Welt ist», «fabulieren» könnte es im Zusammenhang mit Laederachs Schreiben auch heissen, statt «flunkern». Sofern nur jenem wie diesem die unabdingbaren Dimensionen des Ernsthaften und Radikalen zugebilligt werden.

\section{Margrit Baurs «Geschichtenflucht» ${ }^{3}$}

Der schöne Titel signalisiert Weite und Verschachtelung zugleich. Und er bereitet auch schon vor auf ein Erzählen, das über den erzählten Raum, die erzählte Zeit hinausreicht in das der Erzählung nicht mehr Zustehende und Zugänglichedas am Ende aber dennoch sein verborgenster Anlass, seine tiefste Legitimation ist; sein dauernder, unausgesprochener Schmerz auch.

Vier gleichberechtigte Protagonisten ergeben vier Zweierkonstellationen, in denen erzählt wird; auf vier Zeitebenen. Am weitesten zurück liegt das Jahr, das Stefan, damals noch mitten im Berufsund Familienleben stehend, in einem griechischen Dorf verlebt hat und von dem er, Jahrzehnte später, seiner Freundin Helen erzählt. Dies ist die erste Erzählkonstellation. Die zweite ergibt sich dann, wenn Helen dem ihr zuvor unbekannten Studenten und Taxifahrer Etienne erzählt, wie es zu dem früheren Erzählen, dem zwischen Stefan und ihr, gekommen ist, was es ihr bedeutet hat und jetzt, wo Stefan sterbend im Krankenhaus liegt, immer noch bedeuter. In einer dritten Konstellation erzählt Etienne die Geschichte Helens und Stefans seiner Freundin, der Verkäuferin Lisa, weiter; in einer vierten begibt sich diese zu Helen und kommt mit ihrer Version der Geschichte, mit Ergänzungen, Nachträgen, zu Etienne zurück.

Was in der Anlage des Romans nach strenger Geometrie aussieht, ist in der Ausführung die ständige subtile Aufhebung einer solchen. Die Chronologie der Ereignisse ist nicht verpflichtend für die Dramaturgie des Erzählens. Diese gehorcht ganz anderen Gesetzen: dem Gesetz der unmerklichen, oder nur in feinen Rissen, Sprüngen und Brüchen wahrzunehmenden Uebergänge von der einen Erzählkonstellation in die andere. Oder den Gesetzen des Ausspar ens, Ver Schweigens, Sichentziehens, denen zufolge die nicht erzählte Realität zunimmt, je länger das Erzählen dauert, je mehr es nachtragend, ergänzend, erläuternd ein Stück ganze Realität sich anzueignen versucht. Und dies verhift Margot Baurs Erzählen zu den genau austarierten Bewegungen und 
Gegenbewegungen von Annäherung und Flucht, von Festhalten und Loslassen, Verstehen und Nichtverstehen. So wie Etienne es im Zusammenhang mit Lisa erlebt: «Wenn man Lisa zum Thema macht, versickert sie gleich, und was man gepackt hält, hat nicht die mindeste Lisa-Arhnlichkeit. Besser, man denkt an anderes. Da winkt sie manchmal durch ein Hinterfenster herein, und wenn man sie nicht zu halten versucht, bleibt sie ein bisschen da».

Für alle Personen (am ehesten vielleicht nicht für Lisa) sind die Gründe, mit der Entfernung und dem Sichentziehen zu rechnen, weit fassbarer als diejenigen, der Nähe und der Hingabe zu trauen. Und nicht nur vor einander sind sie auf der Flucht, sondern mehr noch vorsieh selber, vor der sie beanspruchenden Realität der Umgebung und der eigenen Empfindungen. Sie durchschauen sich dabei, aber sie offenbaren sich so wenig als möglich. Erst wenn die Kommunikation in besonderem Masse ausgeschlossen erscheint, wagen sie sich ein Stück weit in sie vor.

So Stefan, in der letzten Griechenland-Erfahrung, von der er Helen erzählt: Er ist nach einem langen Winter und nach einer schweren Erkrankung erstmals wieder ins Dorf gegangen, begegnet dabei dem Dorftrottel Kostas und vereint sich mit diesem in einem gespenstischen, grotesken, unendlich hässlichen und zutiefst aufrichtigen Tanz. Kurz danach ist Stefan in die Schweiz zurückgekehrt, nachdem er noch erfahren hatte, dass Kostas am selben Tag gestorben ist. «Er hat sich überfreut», meint, hofft Lisa, wenn sie sich von Etienne die Geschichte erzählen lässt, so wie Etienne sie seinerseits von Helen gehört hat.

Weiter als in dieser Endspiel-Inszenierung von Lebensfreude und Todesnähe ist Margrit Baurs Erzählen vermutlich noch nie gegangen, soviel Emotionalität gegenüber ihren literarischen Figuren und soviel Solidarität mit ihnen gesteht es sich sonst nicht zu. Und es kann nicht Zufall sein, dass die Autorin sich nicht nur emotional, sondern auch sprachlich gerade da derweise exponiert, wo Sprache weitgehend entbehrlich ist, aber an ihre Stelle auch nicht die gefährliche Sprachlosigkeit zwischen die Personen tritt, sondern eine gesteigerte Körperlichkeit und Gestik. Denn für gewöhnlich unterlegt Margrit Baur ihrer Erzählhaltung und -spräche dieselben Fluchtinstinkte, Fluchtreflexe, wie sie ihren Figuren eigen sind. Aus dem Wissen heraus, dass - so formuliert sie es in ihrem Lissaboner Vortrag vom Reden und Schreiben - «in jedem Satz alles schief gehen kann, dass ein einziges Wort das Bild verschiebt». Ihr Schreiben, heisst es da auch, sei der Versuch, «gegen meine Sprachlosigkeit anzugehen».

Und aus der Not dieser Sprachlosigkeit macht Margrit Baur nicht eine Tugend, sondern eine andere Not: eine, die brennt, schmerzt, aber auch: motiviert. Wie bei Georg Büchner, der im März 1934 an seine Braut schreibt: «Ich fürchte mich vor meiner Stimme [...]. Hätte ich einen Weg für mein Inneres; aber ich habe keinen Schrei für den Schmerz; kein Jauchzen für die Freude; keine Harmonie für die Seeligkeit. Dies Stummsein ist meine Verdammnis». In Büchners Klage äussert sich viel von dem, was Margrit Baur am Schreiben hindern würde, wenn sie nicht schriebe. 


\section{NOTES}

1. - Erschienen in: Sonnenaufgänge Sonnenuntergänge, Suhrkamp Verlag, Frankfurt am Main, 1984.

2. - In Vor Schrecken starr. Fixierungen. Stechblicke, Obsessionen, Edition Suhrkamp 1503, Frankfurt-

M. 1987.

3. - Geschichtenfluchl, Suhrkamp Verlag, Frankfurt a.M., 1988. 